\title{
Optimum Short-Term Hydrothermal Scheduling with Spinning Reserve through Network Flows
}

\author{
F.J. Heredia \\ N. Nabona, Member IEEE \\ Statistics \& Operations Research Dept., Universitat Politècnica de Catalunya \\ c. Pau Gargallo 5, 08071 Barcelona, Spain
}

\begin{abstract}
Optimizing the thermal production of electricity in the short term in an integrated power system when a thermal unit commitment has been decided means coordinating hydro and thermal generation in order to obtain the minimum thermal generation costs over the time period under study. Fundamental constraints to be satisfied are the covering of each hourly load and satisfaction of spinning reserve requirements and transmission capacity limits. A nonlinear network flow model with linear side constraints with no decomposition into hydro and thermal subproblems was used to solve the hydrothermal scheduling. Hydrogeneration is linearized with respect to network variables and a novel thermal generation and transmission network is introduced. Computational results are reported.
\end{abstract}

Keywords-Hydrothermal Schednling, Short-Term Operating Planning, Spinning Reserve, Electricity Generation, Nonlinear Network Optimization, Side Constraints

\section{INTRODUCTION}

Short-term hydrothermal coordination is one of the most important problems to be solved in the management of a power utility when hydroelectric plants are a part of the power system. The solution sought indicates how to distribute the hydroelectric generation (cost-free) in each reservoir of the reservoir system and how to allocate generation to thermal units committed to operating over a short period of time (e.g. two days) so that the fuel expenditure during the period is minimized. In shortterm hydrothermal coordination the predicted load at each hourly interval must be met, and a spinning reserve requirement to account for failures or load prediction errors must be satisfied. These load and spinning reserve constraints tie up hydro and thermal generation. As usual, the short term period (of 24 to 168 hours) is subdivided into smaller time intervals (of 1 to 4 hours) for which data are determined and variables are optimized.

Network flow techniques have come to be the most widely used tool for solving this problem. The literature on short-term hydrothermal optimization and coordination through network flows is rich $[1,10]$. The short-term hydrothermal scheduling problem has been researched intensively during recent years, either as the main problem

95 WM 199-0 PWRS A paper recommended and approved by the IEEE Power System Engineering Committee of the IEEE Power Engineering Society for presentation at the 1995 IEEE/PES Winter Meeting, January 29, to February 2, 1995, New York, NY. Manuscript submitted December 29, 1993; made available for printing November 23, 1994.
$[2,3,4]$ or as a subproblem of the short term hydrothermal coordination problem, which includes the commitment of thermal units $[5,6]$. The decoupled method followed by these papers consists in solving the hydro and thermal subproblems separately, coordinating these decoupled optimizations through a) the interchange of the marginal prices of the hourly load demand (from the thermal subproblem to the hydro subproblem) and $b$ ) the hydro generation in each time period (from the hydro subp. to the thermal subp.). In order to solve the hydro subproblems through efficient linear network flow codes, hydrogeneration, which appears in the objective function of the hydro subproblems, is usually approximated as a linear function of the discharges $[2,3]$, or both the discharges and stored water [5]. Quadratic [6] and fully nonlinear formulation [7] of the hydrogeneration has also been reported. The thermal subproblem is usually posed as a set of independent thermal economic dispatch problems if the transmission network is not considered $[5,6]$, or as a set of independent optimal power flow problems, either with a dc $[3,4,7,8]$ or ac $[9]$ approach. The thermal generation and power flows are optimized with a fixed value of the hydrogeneration that corresponds to the optimal solution of the last hydro subproblem. The models proposed in $[2,3,4,6,7]$ take into account the load demands but neglect the spinning reserve, which is included in [5] .

Attempts to solve the hydro and thermal problems together are limited. In [9] a coupled model with an ac OPF solution and a very simplified modeling of the hydro system has been reported.

The decoupled procedure followed in previous works has to assume hydrogeneration values (to define constraints limits) for the thermal minimization and marginal prices of thermal production for hydro optimization. Since both hydrogenerations and marginal prices of thermal generation have unknown values at the optimizer, many solutions to the undecoupled problems will be needed until convergence, which is a clear disadvantage with respect to the undecoupled model.

In a recent work by the authors [11] the network model usually employed for short-term hydrogeneration optimization was extended to include thermal units in an undecoupled way, imposing single load and spinning reserve constraints on both hydro and thermal generators and directly minimizing thermal production costs without decoupling the problem into hydro and thermal subproblems. When constraints are added to limit generation to pre-specified margins at each interval, or to satisfy a given spinning reserve requirement, pure network flow algorithms are no longer applicable. However if these constraints are linearized, efficient specialised algorithms for optimizing network flows with linear side constraints can be employed $[12,13]$.

A specialised nonlinear network flow optimization program with linear side constraints [13] was used to implement the model put forward, and the computational results obtained are reported and compared with the solution provided by a general purpose nonlinear constrained optimization code [15] for the same problem with exact nonlinear hydrogeneration. The linearization of hydrogeneration in terms of the network variables (initial and final 
volumes and discharges at each reservoir) in order to have linear side constraints has proved to yield an acceptable accuracy well within the load prediction errors and with a drastic reduction of execution time.

Transmission constraints and losses can also be taken into account through network flow techniques [10] . In this work they have been integrated into the thermal network developed in [11] .

The undecoupled solution to the short term hydrothermal coordination, taking into account a dc transmission network model, is nothing but a multi-interval dc optimum hydrothermal power flow, where the coupling effects of hydrogeneration over successive intervals are rigorously taken into account and optimized. The classical optimum power flow for a given interval finds values for thermal and for hydrogeneration but requires an "estimated value" of hydrogeneration determined beforehand, and it is not easy to choose values of hydrogeneration over successive intervals so that hydrovariables such as volumes and discharges in reservoir systems are all within limits and match natural inflows. With the undecoupled hydrothermal network model proposed this problem can be readily solved.

\section{Short-Term Hydrogeneration Optimization Through Network Flows}

Fig. 1 represents the well known "replicated" network [1] through which the temporary evolution of the reservoir system is modeled. In Fig. 1 variables $d_{k}^{(i}$ and $s_{k}^{(i}$ stand respectively for the discharge and spillage of reservoir $k$ at time interval $i$, variable $v_{k}^{(i-1}$ is the volume of reservoir $k$ at the beginning of the $i^{\text {th }}$ interval and variable $v_{k}^{(i}$ represents the volume of the same reservoir at the end of the interval, after releasing the discharge $d_{k}^{(i}$ and the spill $s_{k}^{(i}$. The balance equation of the $k^{\text {th }}$ reservoir at the $i^{\text {th }}$ interval would be

$$
a_{k}^{(i}+v_{k}^{(i-1}+d_{k-1}^{(i}+s_{k-1}^{(i}=v_{k}^{(i}+d_{k}^{(i}+s_{k}^{(i}
$$

where $a_{k}^{(i}$ is the natural inflow over the interval in the $k^{\text {th }}$ reservoir.

Network flow algorithms can model any configuration of cascaded hydro stations along branched rivers and water transport delays between successive st.ations. To simplify notation and figures, delays have been omitted in the formulation presented and the terms $d_{k-1}^{i}$ and $s_{k-1}^{(i}$ in the balance equation (1) represent summations of the discharge and spill flows of all upperstream neighboring plants.

The initial and final volumes at each reservoir at each interval and the discharges and spillages at each reservoir over the different intervals will be referred to as the "hydro variables" for they are the flows on the arcs of the replicated hydro network of Fig. 1.

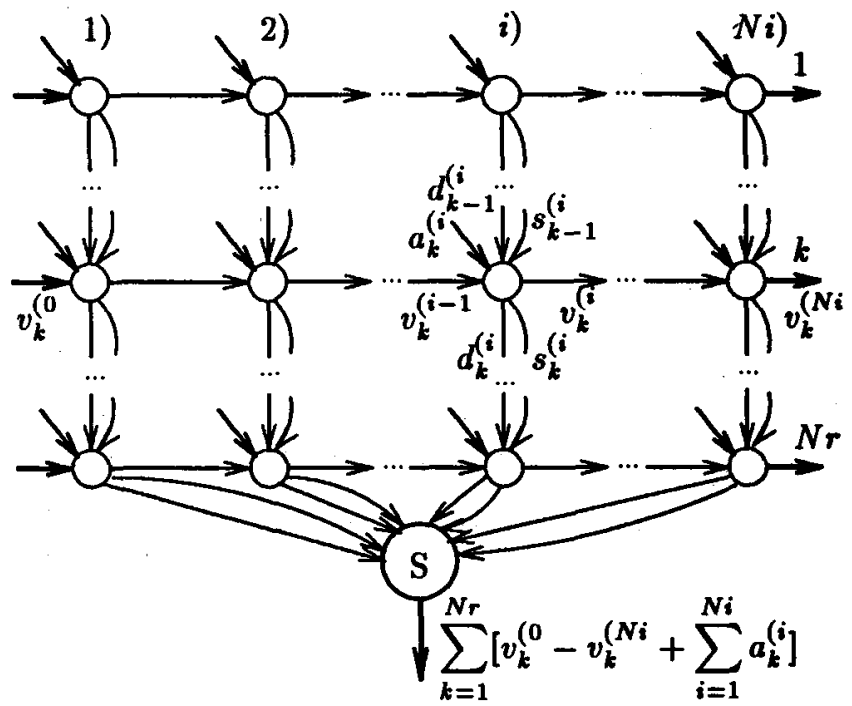

Fig. 1 Replicated network of $N r$ hydrostations and $N i$ intervals

\section{A. Hydrogeneration Function}

In a reservoir system, if the $k^{\text {th }}$ reservoir is of variable head we can compute its generation over the $i^{\text {th }}$ interval as:

$$
H_{k}^{(i}=\mu \rho_{k}{ }^{(i} h_{k}^{(i)} d_{k}^{(i}
$$

where $\mu$ is the mechanical to electrical energy conversion constant and $\rho_{k}{ }^{(i}$ is the efficiency of the $k^{\text {th }}$ reservoir, $h_{k}^{(i)}$ is its equivalent head and $d_{k}{ }^{(i}$ its discharge over the $i^{\text {th }}$ interval. Water head is related to the network variables through a function that gives reservoir head $h$ for stored volume $v$. In the work reported this has been done with a third degree polynomial:

$$
h_{k}=s_{b k}+s_{l k} v_{k}+s_{q k} v_{k}^{2}+s_{c k} v_{k}^{3}
$$

where $s_{b k}, s_{l k}, s_{q k}$ and $s_{c k}$ are the basic, linear, quadratic and cubic shape coefficients of the $k^{\text {th }}$ reservoir. The equivalent head of the $k^{\text {th }}$ reservoir at the $i^{\text {th }}$ interval can be put in terms of the initial and final volume at the $i^{\text {th }}$ interval $v_{k}\left(i-1\right.$ and $v_{k}(i$ :

$$
\begin{gathered}
h_{k}^{(i}=s_{b k}+\frac{s_{l k}}{2}\left(v_{k}^{(i-1}+v_{k}^{(i)}\right)+\frac{s_{q k}}{3}\left(v_{k}^{(i}-v_{k}^{(i-1}\right)^{2} \\
+s_{q k} v_{k}^{(i-1)} v_{k}^{(i}+\frac{s_{c k}}{4}\left[\left(v_{k}^{(i-1)}\right)^{2}+\left(v_{k}^{(i)}\right)^{2}\right]\left(v_{k}^{(i-1)}+v_{k}^{(i)}\right)
\end{gathered}
$$

The efficiency $\rho_{k}(i$ changes with water head and discharge (due to tail-race elevation and other mechanical reasons). It has been modelled as a quadratic function:

$$
\begin{array}{r}
\rho_{k}^{(i}=\rho_{k 0}+\rho_{k h} h_{k}^{(i}+\rho_{k d} d_{k}^{(i}+\rho_{k h d} h_{k}^{(i)} d_{k}^{(i}+ \\
\rho_{k h h}\left(h_{k}^{(i}\right)^{2}+\rho_{k d d}\left(d_{k}^{(i}\right)^{2}
\end{array}
$$

where $\rho_{k 0}, \rho_{k h}, \rho_{k d}, \rho_{k h d}, \rho_{k h h}$ and $\rho_{k d d}$ are efficiency coefficients that must be estimated beforehand. So $h_{k}^{(i}$ is thus modeled as a high order polynomial function of the hydro variables $v_{k}^{(i-1}, v_{k}^{(i}$ and $d_{k}^{(i}$. The hydrogeneration function described is more elaborate than is normal in hydrothermal scheduling $[2,3,6]$ but it leads to a better linearization which produces generation values closer to the real ones, and it does not involve significant extra computation time. 
Assuming that there are $\mathrm{Nr}$ reservoirs, the total hydrogeneration over the $i^{\text {th }}$ interval would be:

$$
H^{(i}=\sum_{k=1}^{N r} H_{k}^{(i}
$$

\section{B. Hydrogeneration Linearization}

Load and spinning reserve constraints will have to be imposed in the optimization process on the total hydrogeneration of each interval. Although these constraints are linear on $H_{k}^{(i}$ they are nonlinear on the hydro variables. In order to ease the optimization effort, these nonlinear constraints are approximated by a linear function of the network variables so that the load and spinning reserve constraints are linear. The linearization used here for $H_{k}^{(i}$ is the one derived from the first order Taylor's series expansion about a former feasible point $\left(v_{F k}^{(i-1}, v_{F k}^{(i}\right.$ and $\left.d_{F k}^{(i}\right)$, which will give an expression such as

$$
H_{L k}^{(i}=\lambda_{0 k}^{(i}+\lambda_{v(i-1) k}^{(i} v_{k}^{(i-1}+\lambda_{v(i) k}^{(i} v_{k}^{(i}+\lambda_{d k}^{(i} d_{k}^{(i}
$$

where $\lambda_{0 k}^{(i}$ is the independent term and $\lambda_{v(i-1) k}^{(i}, \lambda_{v(i) k}^{(i}$ and $\lambda_{d k}^{(i}$ are respectively the linear coefficients of the network variables $v_{k}^{(i-1}, v_{k}^{(i}$ and $d_{k}^{(i}$. The analytical expression of the independent term and of the linear coefficients are easy (though cumbersome) to derive and are given in [11] .

The precision of the linearization described can be judged from the results presented in Sections VIII and IX, where the linearized hydrogeneration results shown satisfy that the sum of thermal generation plus all linearized hydro for a given interval is always within a $\pm 1.5 \%$ margin about the interval's load, which is cuite acceptable given the normal errors in short-term hourly load prediction. This precision will not normally be attained with the first linearization about a feasible point, but just a few linearizations will usually suffice (see Table II). The error incurred in the linearization is measured after an optimum has been obtained. Should the error be above a predetermined tolerance (e.g. $2.0 \%$ of interval's load), a relinearization about the optimum volumes and discharges would be carried out and the problem is then solved again.

\section{Spinning reserve of hydrogeneration}

The expression of the linearized incremental spinning reserve of hydro units (the amount by which the current generation can be increased) in the $i^{\text {th }}$ interval would be $\sum_{k=1}^{N r}\left[\bar{H}_{k}^{(i)}-\left(\lambda_{0 k}^{(i}+\lambda_{v(i-1) k}^{(i} v_{k}^{(i-1}+\lambda_{v(i) k}^{(i} v_{k}^{(i}+\lambda_{d k}^{(i} d_{k}^{(i)}\right)\right]$ where $\bar{H}_{k}^{(i}$ would represent the maximum hydropower of the $k^{\text {th }}$ reservoir over the $i^{\text {th }}$ interval. This maximum generation depends on the actual initial and final volumes $v_{k}^{(i-1}$ and $v_{k}^{(i}$, but in the work reported here it has been precalculated using values $v_{F k}^{(i-1}$ and $v_{F k}^{(i}$ corresponding to a previous feasible point.

The total (linearized) hydrogeneration in the $i^{\text {th }}$ interval $\sum_{k=1}^{N r}\left[\lambda_{0 k}^{(i}+\lambda_{v(i-1) k}^{(i} v_{k}^{(i-1}+\lambda_{v(i) k}^{(i} v_{k}^{(i}+\lambda_{d k}^{(i} d_{k}^{i}\right]$ can be taken as the decremental (linearized) hydro spinning reserve (amount by which the current generation can be decreased) in the interval.

Both the hydro incremental and decremental spinning reserve are assumed to be available within a short (relatively to that of a thermal unit) time lapse

\section{Variables Associated to the Generation OF a Thermal UNIT}

Let $P_{j}$ be the power output of the $j^{\text {th }}$ thermal unit and let $\bar{P}_{j}$ and $\underline{P}_{j}$ be its upper and lower operating limits.

$$
\underline{P}_{j} \leq P_{j} \leq \bar{P}_{j}
$$

The incremental spinning reserve (ISR) $r_{I j}$ of unit " $j$ " is the amount of power by which the current generation $P_{j}$ can be increased within a given time lapse. The maximum possible ISR $\bar{r}_{I j}$ of the $j^{\text {th }}$ unit is the product of the incremental power rate $(\mathrm{MW} / \mathrm{min})$ and the minutes of the specified time lapse. Similarly, the decremental spinning reserve (DSR) $r_{D_{j}}$ of the $j^{\text {th }}$ unit is the amount of power by which one can decrease the current power output $P_{j}$ within a pre-determined time lapse. Its maximum value will be represented by $\bar{r}_{D j}$. The ISR $r_{I j}$ and the DSR $r_{D j}$ of the $j^{\text {th }}$ unit can be expressed as:

$$
\begin{aligned}
r_{I j} & =\min \left\{\bar{r}_{I j}, \bar{P}_{j}-P_{j}\right\} \\
r_{D j} & =\min \left\{\bar{r}_{D j}, P_{j}-\underline{P}_{j}\right\}
\end{aligned}
$$

which is represented by the thick line of Fig. 2a) and $2 b$ ) where a graphical representation of the ISR and the DSR of the $j^{\text {th }}$ unit versus its power output is given.

At power $P_{j}$ we have an ISR $r_{I j}$ and a DSR $r_{D_{j}}$, and there is a power gap $g_{I j} \geq 0$ from the ISR $r_{I j}$ to $\bar{P}_{j}-P_{j}$ and also a power gap $g_{D_{j}} \geq 0$ between the DSR $r_{D_{j}}$ and $P_{j}-\underline{P}_{j}$ so that :

$$
\begin{aligned}
r_{I j}+g_{I j} & =\bar{P}_{j}-P_{j} \\
r_{D j}+g_{D j} & =P_{j}-\underline{P}_{j}
\end{aligned}
$$

\section{A. Network Model of a Single Thermal Unit Generation} and its Spinning Reserve

The generation of a thermal unit, its ISR and DSR, the associated power gaps, and its operating limits lend themselves well to being modeled through network flows. Fig. 2c) and also Fig. 3 show the directed graph having the variables described as flows on its arcs [11].

Node $\mathrm{A}$ has a power injection of $\bar{P}_{j}-\underline{P}_{j}$, which is collected at the sink node $S$. From the balance equations at nodes B and C, equations (11) and (12) are satisfied. Arcs $\alpha$ and $\beta$, both from node $B$ to the sink node $S$, carry the power gap $g_{I j}$ and ISR $r_{I j}$ respectively, and an upper limit of $\bar{r}_{I j}$ on arc $\alpha$ must be imposed to prevent the reserve from getting over its limit. From Fig. 2a) and 2b) it is clear that $r_{I j}$ and its gap $g_{I j}$ must be such that, for a given value of $P_{j}, r_{I j}$ takes the highest value compatible with $r_{I j}<\bar{r}_{I j}$ and with $r_{I j}+g_{I j}=\bar{P}_{j}-P_{j}$. To assure that flows on arcs $\alpha$ and $\beta$ satisfy this, it wolud be enough to place a small positive weighing cost on the flow of arc $\beta$ while arc $\alpha$ has zero cost. The flow $P_{j}-\underline{P}_{j}$ from node $\mathrm{A}$ to node $\mathrm{C}$ is associated to the generation cost.

A network model to represent (9-12) is preferable to extra linear constraints because the efficiency of network codes is higher than that of general purpose linear constraint codes.

In fact the arc going from node $A$ to node $B$ in Fig. 2c) is useless and can be eliminated as in Fig. 3 (since the flow on arc $\alpha$ plus that on arc $\beta$ will amount to $\left.\bar{P}_{j}-P_{j}\right)$. The same happens to be so for the arc going from node $\mathrm{A}$ to node $\mathrm{C}$, which can also be suppressed. However a (generally nonlinear) cost function of its flow $P_{j}-P_{j}$ will have to be optimized, but it suffices to optimize the same function of the sum of flows on arcs $\gamma$ and $\delta$. The simplified thermal network of Fig. 3 can thus be employed. Only for explanatory purposes the notation $P_{j}-\underline{P}_{j}$, equivalent to $r_{D_{j}}+g_{D_{j}}$, will be maintained. 

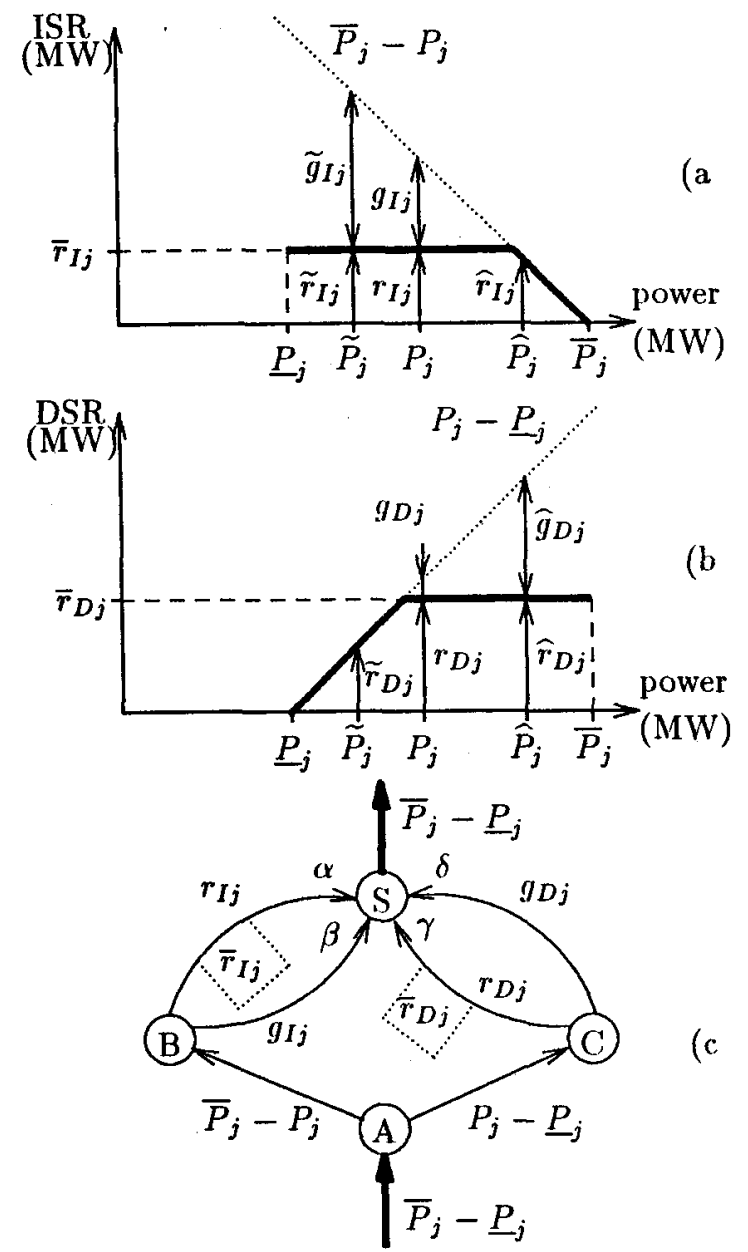

Fig. 2. a) Incremental Spinning Feserve (ISR) function of the $j^{\text {th }}$ thermal unit

b) Decremental Spinning Reserve (DSR) function of the $j^{\text {th }}$ thermal unit

c) Thermal network for the $j^{\text {th }}$ thermal unit indicating limits on arcs $\alpha$ and $\gamma$

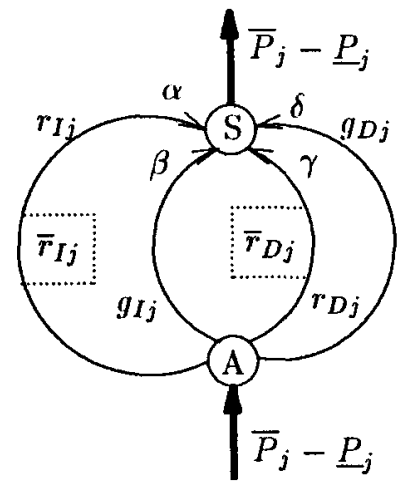

Fig. 3 Equivalent thermal network for the $j^{\text {th }}$ thermal unit supressing nodes $B$ and $C$ and indicating linits on arcs $\alpha$ and $\gamma$.

Although there is no guarantee that the flows on arcs $\gamma$ and $\delta$ are such that flow on arc $\delta$ is as low as possible, it is clear that flow $r_{D_{j}}$ on $\gamma$ will always come to be as high as required to satisfy the minimum DSR constraints imposed. In any case once the optimization is over, the flows on $\operatorname{arcs} \gamma$ and $\delta$ can be redistributed so that $r_{D j}$ is as big as possible with no change in the objective function value.
B. Network Representation of the Ensemble of Thermal Units (Without Transmission Network)

The model just described for one generator can be extended to all committed thermal units at a given interval "i". A single network will represent the generation, ISR, DSR and power gaps of all committed units. The networks. of each single unit can share the sink node $S$ as in Fig. 4 , so the output flow in $\mathrm{S}$ is $\sum_{j=1}^{N u}\left(\bar{P}_{j}-\underline{P}_{j}\right)$ (It can be assumed that for an uncommitted unit at the $i^{\text {th }}$ interval $\bar{P}_{j}=P_{j}^{(i}=\underline{P}_{j}=0$.)

The network described would correspond to the thermal generation and spinning reserve for a single interval "i", and will be referred to as therm.net "i". One such network, connected to a single sink node $\mathrm{S}$, must be considered for each interval. The network balance constraints to be satisfied are:

$$
\begin{gathered}
\bar{P}_{j}-\underline{P}_{j}=\left(\bar{P}_{j}-P_{j}^{(i}\right)+\left(P_{j}^{(i}-\underline{P}_{j}\right)= \\
r_{I j}^{(i}+g_{I j}^{(i}+r_{D j}^{(i}+g_{D j}^{(i}
\end{gathered}=1, \ldots
$$

where the equations correspond to the balance of flow at each node of therm.net " $i$ ".

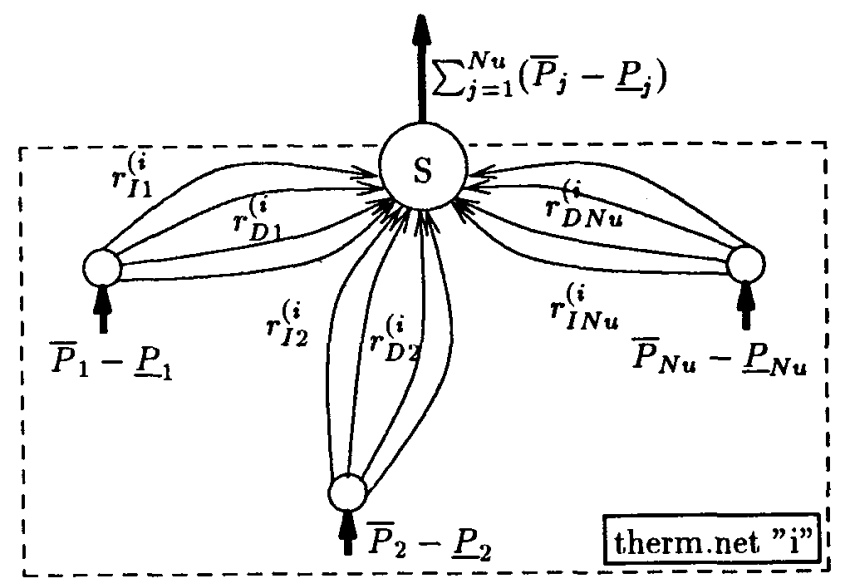

Fig. 4 Network of thermal generation in the $i^{\text {th }}$ interval.

\section{Network Representation of the Ensemble oF Thermal UNITS, HYDROgENERATION and Transmission Network}

Transmission lines with known characteristics and a maximum capacity connect generating units among themselves and to other (load or generating) nodes. The inclusion of the transmission network accounts for transmission limits, which may play an important part in shaping the thermal and hydrogeneration at some intervals.

In what follows it will be shown that it is possible to combine the equivalent network of each thermal unit and a dc model of the transmission network, which take power from hydro and thermal generating stations to the load nodes [10], into a generation plus' transmission network that ensures the satisfaction of load and transmission capacity limits, and where Kirchhoff's current law is satisfied. Kirchhoff's voltage law will also be imposed via linear side constraints on the flows of this network [10]

Node $S$ of Fig. 3 can be split into nodes $T$ and $U$ as in Fig. 5a). It can be noticed that if $\underline{P}_{j}$ is injected in 


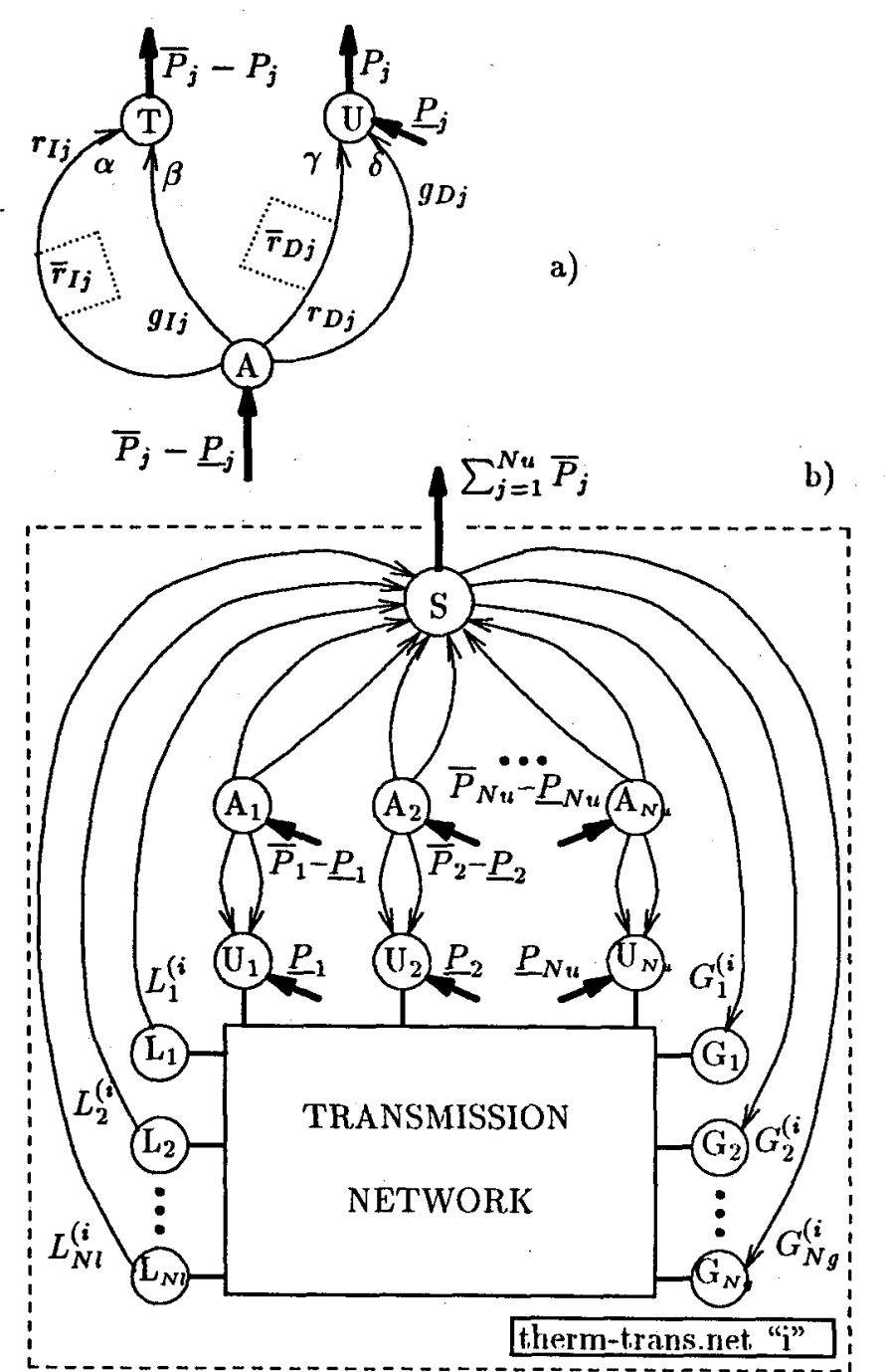

Fig. 5. a) Thermal network for the $j^{\text {th }}$ thermal unit showing node $U$ with power output

b) Thermal network of ensemble of thermal units and connection to transmission network

node $\mathrm{U}$, the outcome of this node will be just $P_{j}$, which is the generation of the $j^{\text {th }}$ thermal unit. The generations of thermal units thus obtained can be fed into a transmission network as in Fig. 5b), where the generation of single reservoirs or of one or more reservoir systems must also be fed (it can be assumed that there are $N g$ nodes where hydrogeneration is fed in). There are one or several $(\mathrm{Nl})$ load nodes in the transmission network and thermal and hydrogeneration must balance the loads which draw from the network.

A sink node $\mathrm{S}$ is used to balance generation and load of a given interval "i". Node $\mathrm{S}$ collects all loads $\left(L_{j}^{(i}, j=1, \ldots, N_{l}\right)$ and supplies hydrogenerations $\left(G_{j}^{(i}\right.$, $\left.j=1, \ldots, N_{g}\right)$ through artificial arcs from the sink node $\mathrm{S}$ to nodes $\mathrm{G}_{j}(j=1, \ldots, N g)$. Nodes $\mathrm{U}_{j},(j=1, \ldots, N u)$ receive the power output $P_{j}^{(i}$, which is fed into the transmission network. All nodes $T$ of the equivalent network of each single thermal unit (see Fig. 5a)) are made to coincide with the sink $\mathrm{S}$. Since the output of node $\mathrm{T}$ is $\bar{P}_{j}-P_{j}^{(i}$ (at interval "i") it is necessary to draw out of node $S$ the flow $\sum_{j=1}^{N u} \bar{P}_{j}$, which is constant, to balance the flow.
Hydrogenerations $H_{k}^{(i}(k=1, \ldots, N r)$ must correspond to the flows on arcs from the sink $\mathrm{S}$ to nodes $\mathrm{G}_{j}(j=1, \ldots, N g)$. In order for this to be so, $N g$ (linear) side constraints employing the linear approximation to hydrogeneration must be imposed:

$$
\begin{array}{r}
G_{j}^{(i}=\sum_{k \in I_{j}}\left[\lambda_{0 k}^{(i}+\lambda_{v(i-1) k}^{(i} v_{k}^{(i-1}+\lambda_{v(i) k}^{(i} v_{k}^{(i}+\lambda_{d k}^{(i} d_{k}^{(i}\right] \\
j=1, \ldots, N g \quad i=1, \ldots, N i
\end{array}
$$

where $I_{j}$ is the set of reservoirs whose hydrogeneration enters into the transmission network through node $\mathrm{G}_{j}$.

The load flow $L_{j}^{(i}$ from node $\mathrm{L}_{j}$ to the sink $\mathrm{S}$ is limited to be within $\pm \epsilon$ of the forecasted load value $l_{j}^{(i}$ at the $i^{\text {th }}$ interval:

$$
l_{j}^{(i}-\epsilon \leq L_{j}^{(i} \leq l_{j}^{(i}+\epsilon
$$

for each interval " $i$ " and load node " $j$ ", $\epsilon$ being an arbitrarily small positive quantity. It would also be possible to place just a negative injection $-l_{j}^{(i}$ at node $\mathrm{L}_{j}$ (for all $j=1, \ldots, N l$ ), but computational experience shows that using extra arcs from $S$ to $L_{j}$ with the $\pm \epsilon$ range considerably eases the task of finding feasible flows; besides, $l_{j}^{(i}$ values are just forecasts subject to prediction errors, and it is therefore natural to allow for a validity range instead of imposing predetermined values.

The transmission network is any set of connections between any of the nodes $\mathrm{U}_{1}, \ldots, \mathrm{U}_{N_{u}}, \mathrm{G}_{1}, \ldots, \mathrm{G}_{N_{g}}, \mathrm{~L}_{1}, \ldots, \mathrm{L}_{N l}$, either directly or via other nodes called transhipment nodes $\left(\mathrm{T}_{1}, \ldots, \mathrm{T}_{N t}\right)$. Taking an arbitrary orientation on each arc of the transmission network, its balance flow equation will be :

$$
\begin{aligned}
& \begin{array}{c}
\sum_{(k, l) \in \mathcal{U}_{j}^{+}} p_{k l}^{(i}-\sum_{(k, l) \in \mathcal{U}_{j}^{-}} p_{k l}^{(i}=P_{j}^{(i} \quad j=1, \ldots, N u \\
\sum p_{k l}^{(i}-\sum p_{k l}^{(i}=G_{j}^{(i} \quad j=1, \ldots, N g
\end{array} \\
& (k, l) \in \mathcal{G}_{j}^{+} \quad(k, l) \in \mathcal{G}_{j}^{-} \\
& \left.\sum p_{k l}^{(i}-\sum p_{k l}^{(i}=L_{j}^{(i} \quad j=1, \ldots, N l\right\} i=1, \ldots, N i \\
& (k, l) \in \mathcal{L}_{j}^{-} \quad(k, l) \in \mathcal{L}_{j}^{+} \\
& \sum p_{k l}^{(i}-\sum p_{k l}^{(i}=0 \quad j=1, \ldots, N t \\
& (k, l) \in \tau_{j}^{+} \quad(k, l) \in \tau_{j}^{-} \\
& -\bar{p}_{k l} \leq p_{k l}^{(i} \leq \bar{p}_{k l} \quad \forall(k, l)
\end{aligned}
$$

Where $\mathcal{U}_{j}^{+}, \mathcal{G}_{j}^{+}, \mathcal{L}_{j}^{+}, \mathcal{T}_{j}^{+}$are the sets of lines that have nodes $U_{j}, G_{j} L_{j}$ and $T_{j}$ as the origin nodes, and $\mathcal{U}_{j}^{-}, \mathcal{G}_{j}^{-}, \mathcal{L}_{j}^{-}, \mathcal{T}_{j}^{-}$the sets of lines with the same nodes as destination nodes. $\bar{p}_{k l}$ is the capacity of the transmission line $(k, l)$.

Since in a dc network model power and current flows measured in per unit (p.u.) coincide, the balance of flow at the nodes of the transmission network ensures the satisfaction of Kirchhoff's current law. Kirchhoff's voltage law must be imposed on all basic loops in the transmission network [10] . Imposing these constraints makes the flows in the transmission network realistic.

Let $x_{k l}$ be the p.u. reactance of the transmission line corresponding to the arc of the equivalent network going from node $k$ to node $l$, and let $p_{k l}$ be the power flow from $k$ to $l$. The voltage drop along arc $k-l$ can then be expressed 
as $x_{k l} p_{k l}$. Thus the expression of Kirchhoff's voltage law is:

$$
\sum_{k, l \in l o o p j} x_{k l} p_{k l}=0 \text { for all basic loops } j
$$

There would be no need of a load-covering constraint because, with the extended network, specific load nodes would receive their share of the total load and specific generation nodes would feed the optimized amount of generated power of its unit. The network of Fig. 5b) will be referred to as therm-trans.net "i".

\section{HYDRo-ThERMAL-TRANSMISSION EXTENDED NETWORK (HTTEN)}

All the variables taking part in the short-term hydrothermal scheduling are flows on arcs of a single network such as that in Fig. 6, called the Hydro-ThermalTransmission Extended Network (HTTEN). A uniçue sink node $S$ collects all the balance water $\sum_{i=1}^{N i} \sum_{k=1}^{N r} a_{k}^{(i}+$ $\sum_{k=1}^{N r}\left(v_{k}^{(0}-v_{k}^{(N i}\right)$ plus the power supplied to the thermal networks $\sum_{i=1}^{N i} \sum_{j=1}^{N u}\left(\bar{P}_{j}-\underline{P}_{j}\right)$ for the case without transmission and $\sum_{i=1}^{N i} \sum_{j=1}^{N u} \bar{P}_{j}$ for the case with transmission. There is no problem in having a common sink node for the replicated hydro network and for the thermal network of each interval because each network is balanced in its own flow. Moreover, all nodes belonging to the hydro network tied to the sink send flow to it and this is so too for the thermal network without transmission. For a thermal network with transmission the only nodes receiving flow from the sink are nodes $\mathrm{G}_{j}(j=1, \ldots, N g)$, which receive the pseudohydrogenerations $G_{j}^{(i}(j=1, \ldots, N g, i=1, \ldots, N i)$, but each of these flows is determined through a side constraint (15).

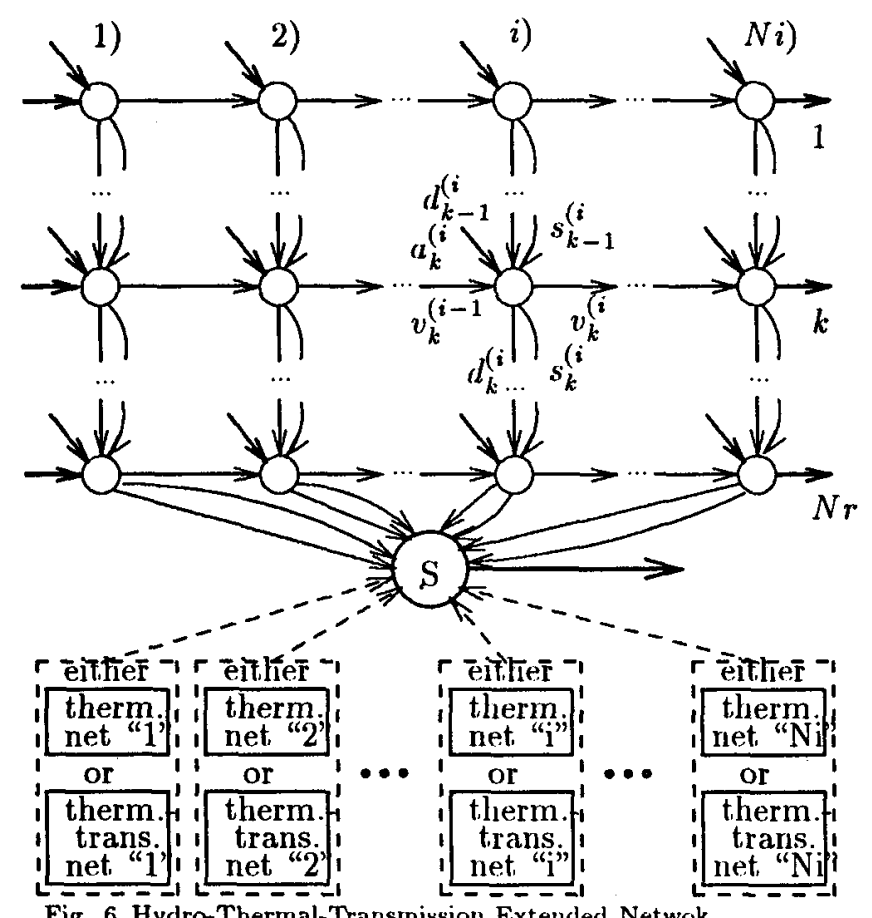

Fig. 6 Hydro-Thermal-Transmission Extended Netwok (HTTEN).
It should be stressed that the fact of using a common sink mode is just a means to reduce the number of balance equality constraints, but it does nor entail that the hydro optimization problem and the thermal optimization problem are coupled. What couples the two problems is the fact that their variables are optimized at the same time with respect to a unique objective function and, most important, subject to common spinning reserve and load constraints where hydro and thermal variables take part.

\section{Generation cost of THERMaL UNITS AND} LOSSES IN THE TRANSMISSION NETWORK

The production cost of the $j^{\text {th }}$ thermal unit over the $i^{\text {th }}$ interval, expressed as a second order polynomial with a linear and a quadratic cost coefficient $c_{i j}$ and $c_{q j}$ would be $c_{l j} P_{j}^{(i}+c_{q j}\left(P_{j}^{(i}\right)^{2}$, which in terms of the network flow $P_{j}^{(i}-\underline{P}_{j}=r_{D j}+g_{D j}$ is $\left(c_{l j}+2 c_{q j} \underline{P}_{j}\right)\left(P_{j}^{(i}-\underline{P}_{j}\right)+c_{q j}\left(P_{j}^{(i}-\right.$ $\left.\underline{P}_{j}\right)^{2}+\left(c_{l j} \underline{P}_{j}+c_{q j} \underline{P}_{j}{ }^{2}\right)$. The last parenthesis is of constant terms and can be excluded from the minimization. So the expression to be minimized is: $\left(c_{l j}+2 c_{q j} \underline{P}_{j}\right)\left(r_{D j}^{(i}+g_{D j}^{(i}\right)+$ $c_{q j}\left(r_{D j}^{(i}+g_{D j}^{(i}\right)^{2}$. Thus the thermal part of the cost function (corresponding to the $i^{\text {th }}$ interval) to be minimized can be expressed as:

$$
\min \sum_{j=1}^{N u}\left[\left(c_{l j}+2 c_{q j} \underline{P}_{j}\right)\left(r_{D j}^{(i}+g_{D j}^{(i}\right)+c_{q j}\left(r_{D j}^{(i}+g_{D j}^{(i}\right)^{2}\right]
$$

When the equivalent network model of Section IV is considered, the transmission network model is a dc approach and losses are not included in the generation injected. Power losses can be evaluated and added to the objective function to be minimized. $p_{k l}^{(i}$ being the p.u. value of power flow on the arc from node $k$ to node $l$ at the $i^{\text {th }}$ interval, $r_{k l}$ being the p.u. resistence of the transmission line corresponding to the arc, the losses on that line are $r_{k l}\left[p_{k l}^{(i}\right]^{2}$, and $\pi^{(i}$ being a price given to the losses over the $i^{\text {th }}$ interval, the term to be added to the objective function would be:

$$
\sum_{i=1}^{N i} \pi^{(i} \sum_{k, l \in \mathrm{T}: \mathrm{N} .} r_{k l}\left[p_{k l}^{(i}\right]^{2}
$$

where T.N. is the set of pairs of nodes that are the ends of all transmission lines.

\section{UNDECOUPLED NETWORK FORMUlation OF THE Hydro-Thermal Scheduling}

\section{A. Objective Function.}

The objective function to be minimized is either $\min \sum_{i=1}^{N i}\left\{\sum_{j=1}^{N u}\left[\left(c_{l j}+2 c_{q j} \underline{P}_{j}\right)\left(P_{j}^{(i}-\underline{P}_{j}\right)+c_{q j}\left(P_{j}^{(i}-\underline{P}_{j}\right)^{2}\right]\right\}$

without transmission network, or

$$
\begin{gathered}
\min \sum_{i=1}^{N i}\left\{\sum _ { j = 1 } ^ { N u } \left[\left(c_{l j}+2 c_{q j} \underline{P}_{j}\right)\left(P_{j}^{(i}-\underline{P}_{j}\right)+\right.\right. \\
\left.\left.c_{q j}\left(P_{j}^{(i}-\underline{P}_{j}\right)^{2}\right]+\pi^{(i} \sum_{k, l \in \mathrm{T} . \mathrm{N} .} r_{k l}\left[p_{k l}^{(i}\right]^{2}\right\}
\end{gathered}
$$

with transmission network, where the last term corresponds to the evaluation of the losses. 


\section{B. Network Constraints.}

The network constraints for hydro-variables and thermal variables are

$$
\begin{array}{r}
a_{k}^{(i}+v_{k}^{(i-1}+d_{k-1}^{(i}+s_{k-1}^{(i}=v_{k}^{(i}+d_{k}^{(i}+s_{k}^{(i} \quad k=1, \ldots, N r \\
i=1, \ldots, N i \\
\bar{P}_{j}-\underline{P}_{j}=r_{I j}^{(i}+g_{I j}^{(i}+r_{D j}^{(i}+g_{D j}^{(i} \quad j=1, \ldots, N u \\
i=1, \ldots, N i
\end{array}
$$

The balance network constraints for the transmission network are those expressed in (17). The balance equation at the sink node $\mathrm{S}$ when no transmission network is considered would be:

$$
\begin{gathered}
\sum_{i=1}^{N i}\left\{d_{N r}^{(i}+s_{N r}^{(i}+\sum_{j=1}^{N u}\left(r_{I j}^{(i}+g_{I j}^{(i}+r_{D j}^{(i}+g_{D j}^{(i}\right)\right\}= \\
\sum_{i=1}^{N i}\left\{\sum_{k=1}^{N r} a_{k}^{(i}+\sum_{k=1}^{N r}\left(v_{k}^{(0)}-v_{k}^{(N i}\right)+\sum_{j=1}^{N u}\left(\bar{P}_{j}-\underline{P}_{j}\right)\right\}
\end{gathered}
$$

and should the transmission network be included the balance at $\mathrm{S}$ would be:

$$
\begin{array}{r}
\sum_{i=1}^{N i}\left\{d_{N r}^{(i}+s_{N r}^{(i}+\sum_{j=1}^{N u}\left(r_{I j}^{(i}+g_{I j}^{(i)}\right)-\sum_{j=1}^{N g} G_{j}^{(i}+\sum_{j=1}^{N l} L_{j}^{(i}\right\}= \\
\sum_{i=1}^{N i}\left\{\sum_{k=1}^{N r} a_{k}^{(i}+\sum_{k=1}^{N r}\left(v_{k}^{(0)}-v_{k}^{(N i}\right)+\sum_{j=1}^{N u} \bar{P}_{j}\right\}
\end{array}
$$

Upper and lower limits, which are zero for most of the variables, exist for all the flows. They are taken into account by the specialised network codes.

\section{Load and Spinning Reserve Coupling Constraints.}

Side constraints [12] (i.e.: constraints on the flows on the arcs different from the flow balance equations at each node) can be imposed and can be dealt with efficiently in specific network flow optimization methods [12,13]. Such side constraints could be a load constraint, so that (at each interval) a given load $L$ is met by the thermal units plus hydro units output, and minimum ISR and DSR requirements $R_{I}$ and $R_{D}$ to be satisfied.

If no transmission network is considered, it is necessary to add up the minimum power output $P j$ of thermal unit $j$ over the $i^{\text {th }}$ interval to the sum of flows $r_{D j}^{(i}+g_{D j}^{(i}=$ $P_{j}^{(i}+\bar{P} j$ to get $P_{j}^{(i}$. Thus the constraints to ensure that load $L^{(i}$ is met at the $i^{\text {th }}$ interval can be cast as

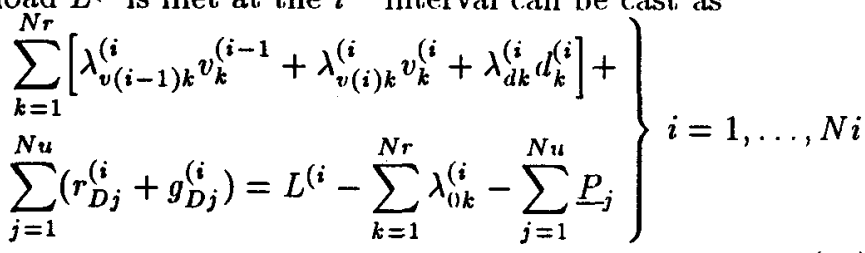

Should a transmission network be considered throngh the equivalent thermal plus transmission network presented Section IV, there is no need for a specific load constraint as (27) because the transmission network balance equations (17) ensure the satisfaction of load at each interval. Instead, the hydrogeneration side constraints (15) and Kirchhoff's voltage law sicle constraints (18) must be imposed.

The satisfaction of the incremental and decremental spinning reserve requirements at each interval:

$$
\left.\begin{array}{r}
-\sum_{k=1}^{N r}\left[\lambda_{v(i-1) k}^{(i} v_{k}^{(i-1)}+\lambda_{v(i) k}^{(i} v_{k}^{(i}+\lambda_{d k}^{(i} d_{k}^{(i}\right]+ \\
+\sum_{j=1}^{N u} r_{I j}^{(i} \geq R_{I}^{(i}-\sum_{k=1}^{N r} \bar{H}_{k}^{(i}+\sum_{k=1}^{N r} \lambda_{0 k}^{(i} \\
\sum_{k=1}^{N r}\left[\lambda_{v(i-1) k}^{(i} v_{k}^{(i-1)}+\lambda_{v(i) k}^{(i} v_{k}^{(i}+\lambda_{d k}^{(i} d_{k}^{(i}\right]+ \\
+\sum_{j=1}^{N u} r_{D j}^{(i} \geq R_{D}^{(i}-\sum_{k=1}^{N r} \lambda_{0 k}^{(i}
\end{array}\right\}
$$

These load and ISR and DSR constraints constitute the coupling between the hydro and the thermal network of each interval. The replicated hydro network involves a coupling between the hydro and the thermal variables of all intervals.

\section{Computational Results and Case Example}

The network model put forward has been implemented to solve hydrothermal scheduling problems. The code used, NOXCB [14], is a specialised nonlinear network flow program with linear side constraints. The code has been developed in Fortran 77 and has been used to solve the set of case examples described in Table I on a Sun Sparc 10/41 workstation.

Case examples of type A (problems A24, A48, and A168) correspond to reservoir system 1 of Tables III to V. Case examples of type B (problems B48 and B168) correspond to a composite reservoir system made of reservoir systems 1 and 2 of Tables III to V. Cases A and B have the thermal power system of Table VI. Problems A24 to B168 have been solved without transmission network while problems $\mathrm{B} 48 \mathrm{x}$ and $\mathrm{B} 168 \mathrm{x}$ consider the 5-bus 6-line transmission network described in Fig. 8 and Table VII. Case examples of type $\mathrm{C}$ correspond to a bigger power system whose data are not included in this paper, but which can be made available upon request.

Table I : Case examples

\begin{tabular}{|c|c|c|c|c|c|c|c|c|}
\hline \multirow{2}{*}{$\begin{array}{c}\text { Problem } \\
\text { ident. }\end{array}$} & \multicolumn{4}{|c|}{ Power system size } & \multicolumn{3}{c|}{ HTTEN size } \\
\cline { 2 - 8 } & $N r$ & $N u$ & $N m$ & $N b$ & $N i$ & arcs & nodes & S.C. \\
\hline A24 & 3 & 4 & - & - & 24 & 648 & 163 & 72 \\
A48 & 3 & 4 & - & - & 48 & 1248 & 313 & 144 \\
A168 & 3 & 4 & - & - & 168 & 4536 & 1135 & 504 \\
\hline B48 & 6 & 4 & - & - & 48 & 1824 & 457 & 144 \\
B168 & 6 & 4 & - & - & 168 & 6552 & 1639 & 504 \\
\hline B48x & 6 & 4 & 6 & 5 & 48 & 2256 & 697 & 240 \\
B168x & 6 & 4 & 6 & 5 & 168 & 8064 & 2479 & 840 \\
\hline C48 & 9 & 8 & 19 & 12 & 48 & 4416 & 1346 & 528 \\
\hline
\end{tabular}

Figs. 7, 9 and 10 illustrate the results of case example $\mathrm{B} 48 \mathrm{x}$. There are 3 cascaded reservoirs in each reservoir system $(N r=2 \times 3=6)$, which will be referred to as "upper", "middlle" and "lower" reservoir, 4 thermal units $(N u=4)$, a 5 -bus $(N b=5) 6$-line $(N m=6)$ transmission network with two load busses $(N l=2)$, two basic loops and 48 one-hour intervals $(N i=48)$. Thermal units Th1 and Th2 are connected to the same bus and so are Th3 and Th4. The hydrogeneration of the composite reservoir system is injected to another bus. The resulting HTTEN has 2256 arcs (variables), 697 nodes (network balance equations) and $48 \times(3+2)=240$ side constraints. 


\section{Load (MW)}

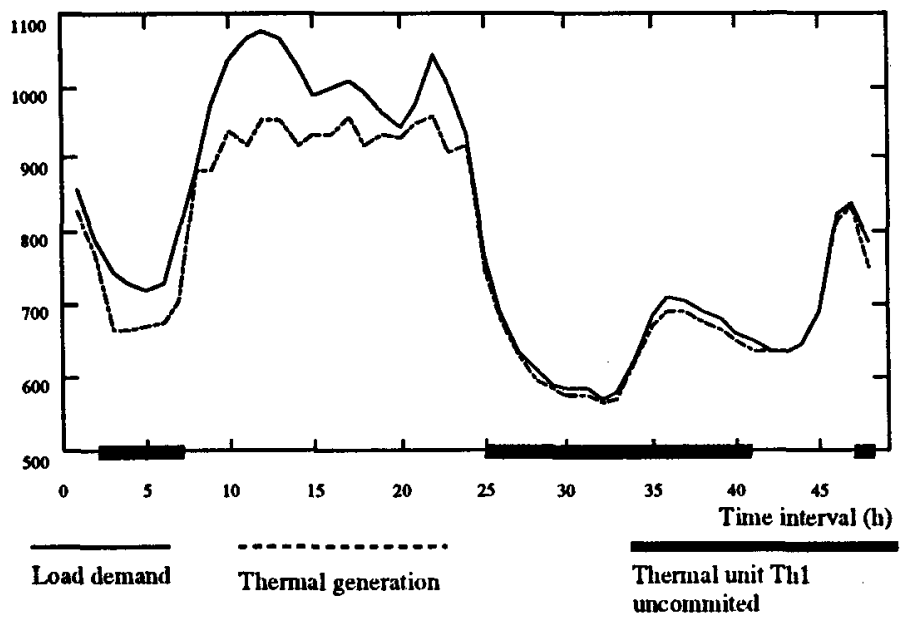

Fig. 7 Atteintment of load at the optimal solution of case B48x.

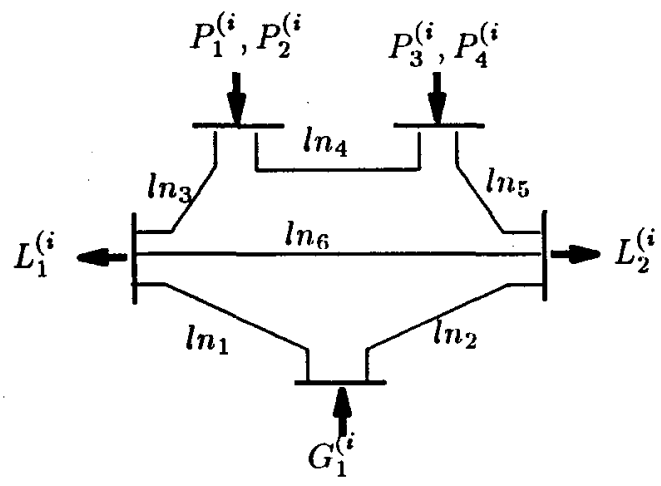

Fig. 8 Transmission network of case $B 48 x$.

Thermal unit Th1 is uncommited from intervals 2 through 7 , from intervals 25 through 41 and at intervals 47 and 48 . Thermal units Th2, Th3 and Th4 are operating throughout the entire period. The ISR constraint considered is a 7 minute one and its requirement is $R_{I}=450 \mathrm{MW}$ for all intervals. A 5 minute DSR constraint has been considered for each interval. The DSR requirements considered were $15 \%$ of interval forecasted load, (thus $\left.R_{D}^{(i}=.15 \times\left(L_{1}^{(i}+L_{2}^{(i}\right)\right)$. Initial and final volumes of reservoirs are the same and correspond to $3 / 4$ of the maximum volume $\left(v_{k}^{(0)}=3 / 4 \bar{v}_{k}, v_{k}^{(48} \geq 3 / 4 \bar{v}_{k}, k=1, \ldots, 5\right)$, except for the lower reservoir of reservoir system 2, which is held fixed to maximum volume $\left(v_{6}^{(i}=\bar{v}_{k}, \forall i\right)$.

The first point employed to compute the hydro linearization coefficients and the maximum hydrogenerations (to be used in the ISR constraints) corresponds to constant maximum volumes with discharges that maximize hidrogeneration. The optimum obtained after three linearizations has a mismatch of linearized to exact hydrogeneration below $1.3 \%$ of interval load, that is :

$$
\left|H_{k}^{(i}-H_{L k}^{(i}\right| \leq 0.013\left(L_{1}^{(i}+L_{2}^{(i}\right) \quad i=1, \ldots, N i
$$

The load constraints (see Fig. 7) are thus matched with a maximum error of $1.3 \%$ of forecast load (at interval 4) and the ISR and DSR constraints are satisfied (see Fig. 9 and 10).The total time re(puired was $49.5 \mathrm{~s}$ of CPU time. It must be stressed that the high value of the hydrogeneration during intervals 2 through 7 is due to thermal unit Th1 being uncommited. The ISR constraint is active at intervals $7,11,12$ and 48 while the DSR is active from intervals 24 through 41 , and at intervals $2,8,20$ and 21 . The transmission capacity limit of line $\ln _{3}$ is active at intervals $12,13,17$ and 22 .

\section{ISR (MW)}

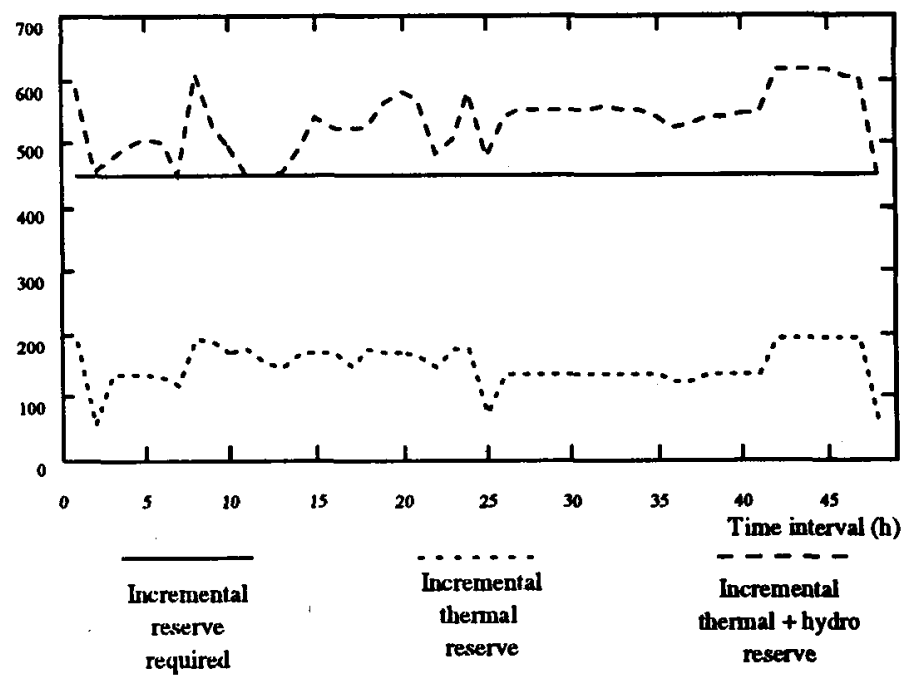

Fig. 9 Incremental reserve at the optimal solution of case B48x.

\section{DSR (MW)}

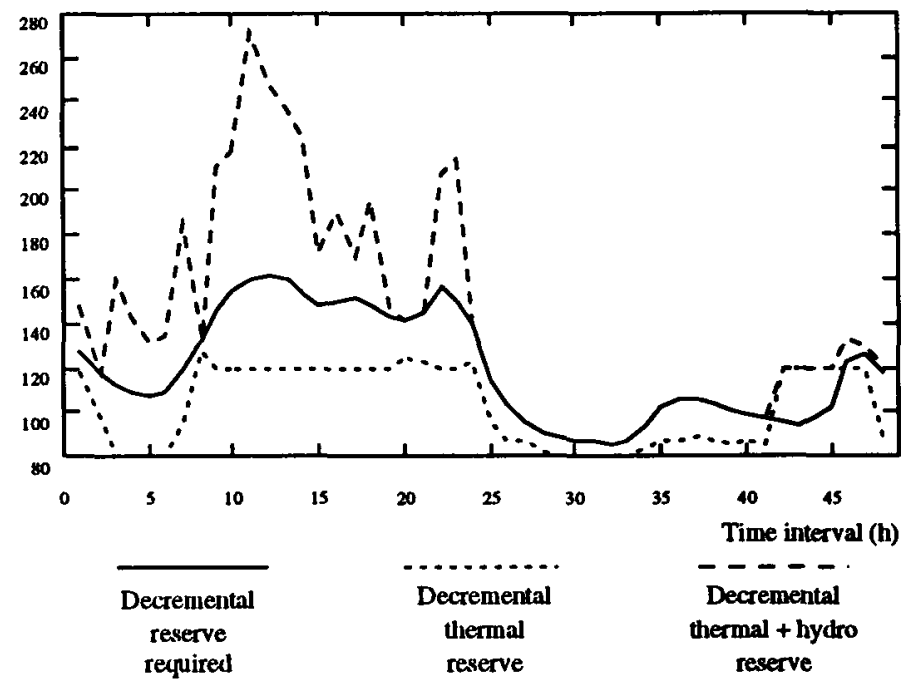

Fig. 10 Decremental reserve at the optimal solution of case B48x.

\section{Results Obtained Using a General Purpose NONLINEAR CODE}

The general purpose nonlinear optimization code $\mathrm{MI}$ NOS $5.3[15,16]$ has been used to solve the same test problem. A change introduced in the formulation when using the MINOS code has been not to linearize hydrogeneration so that the genuine hydrogeneration $H_{k}^{(i}=\mu \rho_{k}{ }^{i} h_{k}{ }^{(i} d_{k}{ }^{(i}$ taking into account (3) and (5) is employed instead of $\lambda_{0 k}^{(i}+\lambda_{v(i-1) k}^{(i} v_{k}^{(i-1}+\lambda_{v(i) k}^{(i} v_{k}^{(i}+\lambda_{d k}^{(i} d_{k}^{(i}$ in (27) and (28).

The results obtained are shown in Table II, where the solution values and the computation times can be compared to those obtained using the NOXCB code 
Table V : Efficiency of the hydrogeneration

linearizing hydrogenerations. The purpose of Table II is not to compare the efficiency of the two codes, because NOXCB and MINOS are here used to solve different formulations of the same problem. Instead, Table II can be used to evaluate the suitability (with respect to CPU time and solution precision) of using an approximated linear formulation, which leads to faster execution times and realistic values of the cost function, but which gives solutions that admit a violation of load and reserve constraints up to the maximum generation error fixed by the user.

Table II : Computational results

\begin{tabular}{|c|c|c|c|c|c|c|}
\hline Problem & max. & no. of & \multicolumn{2}{|c|}{ CPU (8) } & \multicolumn{2}{c|}{ Cost (10 $0^{6}$ Pts) } \\
\cline { 4 - 7 } ident. & gen. err. & linear. & NO. & MI. & NO. & MI. \\
\hline A24 & $\leq 0.7 \%$ & 3 & 14.7 & 38.7 & 73.10 & 73.15 \\
A48 & $\leq 1.1 \%$ & 3 & 39.2 & 219.6 & 124.23 & 124.39 \\
A168 & $\leq 1.4 \%$ & 3 & 623.5 & 6530.7 & 361.82 & 362.16 \\
\hline B48 & $\leq 0.9 \%$ & 3 & 31.2 & 514.3 & 123.07 & 123.19 \\
B168 & $\leq 1.5 \%$ & 2 & 336.2 & 6667.8 & 361.30 & 361.62 \\
\hline B48x & $\leq 1.3 \%$ & 3 & 49.5 & 394.1 & 132.15 & 132.34 \\
B168x & $\leq 1.4 \%$ & 2 & 538.4 & 4963.2 & 384.40 & 384.54 \\
\hline C48 & $\leq 1.5 \%$ & 2 & 337.6 & 6020.1 & 199.32 & 199.47 \\
\hline
\end{tabular}

\section{A. Noncovexity of the Constraints.}

The formulation proposed for the problem, with linearized hydrogeneration, is that of minimizing (21) or (22) subject to only linear constraints. However, when a nonlinear hydrogeneration function is considered, as has been done with MINOS, some of the constraints are nonlinear and some of them are not convex. (It can be noticed in (28) that the ISR requirement has $-H_{k}^{(i}$ in it, whereas the DSR requirement has $+H_{k}^{(i)}$ so that one or the other is nonconvex). In spite of this the computational experience shows that the effects of nonconvexity are not important, as the results obtained with the specialised network code NOXCB, with a convexified problem, very much resemble those obtained with the general code MINOS with the real nonconvex problem.

Table III : Characteristics of the hydro system

\begin{tabular}{|c|c|c|c|c|}
\hline Res.S.1 & $\begin{array}{c}\text { Max./Min. } \\
\text { vol. } \\
\left(\mathrm{Hm}^{3}\right)\end{array}$ & $\begin{array}{l}\text { Nat. } \\
\text { inflow } \\
\left(\mathrm{m}^{3} / \mathrm{s}\right)\end{array}$ & $\begin{array}{c}\text { Num. } \\
\text { of } \\
\text { disch. }\end{array}$ & $\begin{array}{l}\text { Max. } \\
\text { disch. } \\
\left(\mathrm{m}^{3} / \mathrm{s}\right)\end{array}$ \\
\hline $\begin{array}{l}\text { Upper } \\
\text { Middle } \\
\text { Lower }\end{array}$ & $\begin{array}{l}1340.0 / 0 \\
136.0 / 0 \\
160.0 / 0\end{array}$ & $\begin{array}{l}25.0 \\
10.0 \\
5.0\end{array}$ & $\begin{array}{l}2 \\
2 \\
2\end{array}$ & $\begin{array}{l}320.0 \\
440.0 \\
80.0\end{array}$ \\
\hline $\begin{array}{l}\text { Upper } \\
\text { Middle } \\
\text { Lower }\end{array}$ & $\begin{array}{l}354.0 / 0 . \\
160.0 / 0 . \\
2.0 / 2.0\end{array}$ & $\begin{array}{l}2.5 \\
2.0 \\
1.0\end{array}$ & $\begin{array}{l}2 \\
2 \\
2\end{array}$ & $\begin{array}{l}60.0 \\
40.0 \\
20.0\end{array}$ \\
\hline
\end{tabular}

Table IV : Reservoir head to volume coefficients

\begin{tabular}{|c|c|c|c|c|}
\hline Res.5.1 & \multicolumn{4}{|c|}{ Res. head : $h_{k}=s_{b k}+s_{1 k} v_{k}+s_{q k} v_{k}{ }^{2}+s_{c k} v_{k}{ }^{3}$} \\
\hline Res.S.2 & $\begin{array}{l}s_{b k} \\
(m)\end{array}$ & $\begin{array}{c}s_{l k} \\
\left(\mathrm{~m} / \mathrm{Hm}^{3}\right)\end{array}$ & $\begin{array}{c}s_{q k} \\
\left(m / H_{n^{6}}\right)\end{array}$ & $\begin{array}{c}s_{c k} \\
\left(\mathrm{~m} / \mathrm{H}^{\mathrm{g}}\right)\end{array}$ \\
\hline Upper & 30.419 & 0.04159999 & $-.224878210^{-4}$ & $0.641298110^{-8}$ \\
\hline Middle & 19.00889 & 0.09927949 & $-.261145310^{-3}$ & $0.528149010^{-0}$ \\
\hline Lower & 12.0 & 0.1169008 & $-.193817310^{-3}$ & $0.309578610^{-6}$ \\
\hline Upper & 40.5835 & 0.1914066 & $-.430230810^{-3}$ & $0.485060410^{-6}$ \\
\hline Middle & 12.0 & 0.1160998 & $-.193817310^{-3}$ & $0.309578610^{-6}$ \\
\hline Lower & 79.0 & 0.00000000 & 0.0 & 0.0 \\
\hline
\end{tabular}

\begin{tabular}{|c|c|c|c|}
\hline Res.S.1 & $\rho_{0}$ & $\begin{array}{c}\rho_{h d} \\
\rho_{h h}\end{array}$ & $\begin{array}{c}\rho_{h} \\
\end{array}$ \\
\hline Upper & $\rho_{d}$ & $\rho_{d d}$ \\
& -.21311 & $0.4510^{-4}$ & $0.2276210^{-1}$ \\
& $0.932910^{-2}$ & $-.2910^{-3}$ & $-0.410^{-1}$ \\
\hline Middle d1 & 0.4747001 & $0.845710^{-5}$ & $0.209746510^{-1}$ \\
& $0.178491610^{-2}$ & $-.36657810^{-3}$ & $-.660510^{-5}$ \\
\hline Middle d2 & 0.4870272 & $-.157710^{-4}$ & $0.179933710^{-1}$ \\
& $0.204303710^{-2}$ & $-.24117310^{-3}$ & $-.469810^{-5}$ \\
\hline Lower & 0.4375 & $-0.991900010^{-5}$ & $0.187087710^{-1}$ \\
& $0.163148210^{-1}$ & $-0.337316010^{-3}$ & $-0.275372010^{-3}$ \\
\hline Res.S.2 & $\rho_{0}$ & $\rho_{h d}$ & $\rho_{h}$ \\
& $\rho_{d}$ & $\rho_{h h}$ & $\rho_{d d}$ \\
\hline Upper & 0.113 & $0.128231010^{-4}$ & $0.988461610^{-2}$ \\
& $0.164697510^{-1}$ & $-0.749190010^{-4}$ & $-0.749190010^{-4}$ \\
\hline Middle & 0.4375 & $-0.991900010^{-8}$ & $0.187087710^{-1}$ \\
& $0.163148210^{-1}$ & $-0.337316010^{-3}$ & $-0.275372010^{-3}$ \\
\hline Lower & 0.2695 & 0.0 & 0.0 \\
& $0.768526210^{-1}$ & 0.0 & $-0.225862810^{-2}$ \\
\hline
\end{tabular}

Table VI : Thermal units

\begin{tabular}{|c|c|c|c|}
\hline & & Incr. rate & Production cost \\
\hline Unit & $\frac{P}{(\mathrm{MW})}$ & $\begin{array}{l}\text { Decr. rate } \\
\text { (MW/min) }\end{array}$ & $\begin{array}{c}c_{l}(\mathrm{Pts} / \mathrm{MWh}) \\
c_{q}\left(\mathrm{Pts} /(\mathrm{MWh})^{2}\right)\end{array}$ \\
\hline Th1 & $\begin{array}{l}160.0 \\
80.0\end{array}$ & $\begin{array}{l}3.5 \\
3.5\end{array}$ & $\begin{array}{c}2121.5168 \\
9.639808\end{array}$ \\
\hline Th2 & $\begin{array}{l}350.0 \\
100.0\end{array}$ & $\begin{array}{l}8.0 \\
8.0\end{array}$ & $\begin{array}{c}3173.0382 \\
0.833415\end{array}$ \\
\hline Th3 & $\begin{array}{l}350.0 \\
100.0\end{array}$ & $\begin{array}{l}8.0 \\
8.0\end{array}$ & $\begin{array}{c}3228.7386 \\
0.848045\end{array}$ \\
\hline Th4 & $\begin{array}{l}350.0 \\
100.0\end{array}$ & $\begin{array}{l}8.0 \\
8.0\end{array}$ & $\begin{array}{c}3152.0982 \\
0.827915\end{array}$ \\
\hline
\end{tabular}

Table VII : Transmission line data

\begin{tabular}{|c|c|c|c|c|c|}
\hline Line & kV & km & $\begin{array}{c}R \\
(\Omega / \mathbf{k m})\end{array}$ & $\begin{array}{c}X \\
(\Omega / \mathbf{k m})\end{array}$ & $\begin{array}{c}\text { Capacity } \\
\text { (A) }\end{array}$ \\
\hline $\ln _{2}$ & 230 & 108.79 & 0.088 & 0.4654 & 900 \\
\hline $\ln$ & 23 & 63.0 & 0.044 & 0.2327 & 1800 \\
\hline $\ln _{3}$ & 230 & 108.79 & 0.088 & 0.4654 & 900 \\
\hline $\ln _{4}$ & 230 & 18.45 & 0.044 & 0.2327 & 1800 \\
\hline $\ln s$ & 230 & 55.5 & 0.022 & 0.11635 & 3600 \\
\hline $\ln _{G}$ & 230 & 31.8 & 0.176 & 0.9308 & 1800 \\
\hline
\end{tabular}

Table VIII : Forecasted load

\begin{tabular}{|c|c|c|c|c|c|c|c|c|}
\hline & $\begin{array}{c}L_{1}^{(i} \\
(\mathrm{MW})\end{array}$ & $\begin{array}{c}L_{2}^{(i} \\
(\mathrm{MW})\end{array}$ & $(i$ & $\begin{array}{c}L_{1}^{(i} \\
(\mathrm{MW})\end{array}$ & $\begin{array}{c}L_{2}^{(i)} \\
(\mathrm{MW})\end{array}$ & $\begin{array}{c}L_{1}^{(i} \\
(i\end{array}$ & $\begin{array}{c}L_{2}^{(i)} \\
(\mathrm{MW})\end{array}$ \\
$(\mathrm{MW})$
\end{tabular}




\section{Conclusions}

An undecoupled formulation of the optimal short-term hydro-thermal scheduling featuring a new thermal unit network model has been presented and demonstrated. The results obtained indicate that the solution to this problem is possible and that the computation resources required are moderate. The undecoupled formulation is more advantageous than the decoupled one because a single optimization leads to the optimum and there is no need to repeat optimizations with updated estimations of the Lagrange multipliers or of hydrogenerations, which could not converge on the optimum of the problem.

The linearization of hydrogeneration with respect to initial and final volume and discharge at each interval produces results of sufficient accuracy and permits the use of specialised network flow codes, with linear side constraints, which are much more efficient than general purpose nonlinear optimization codes and prove to be an excellent tool for hydrothermal scheduling.

\section{Acknowledgment.}

The collaboration of the student. Alex Chiva Blay in the preparation of the solutions with MINOS is gratefully acknowleged.

\section{REFERENCES}

[1] Rosenthal, R.E. "A Nonlinear Network Flow Algorithm for Maximization of Benefits in a Hydroelectric Power System ". Operations Research, vol. 29, no. 4,1981 , pp. $763-784$.

[2] Luo, G.X., H. Habibollahzadeh, A. Semlyen. "ShortTerm Hydro-Thermal Dispatch Detailed Model and Solutions". IEEE Trans. on Power Systems, vol. 4, no. 4, November 1989 , pp. 1452-1462.

[3] Johannesen, A., A. Gjelsvik, O.B. Fosso, N. Flatab $\varnothing$, "Optimal Short-Term Hydroelectric Scheduling Including Security Constraints". IEEE Trans. on Power Systems, vol. 6, no. 2, May 1991, pp. 576583.

[4] Ohishi, T., S. Soares, W.F.H. Carvalho. "Shortterm Hydrothermal Scheduling Alproach for Predominantly Hydroelectric Systems". IEEE Trans. on Power Systems, vol.6, no.2, May 19!1, pp. 637643.

[5] Li, Chao-an, P.J. Jap, D.L. Streiffert, "Implementation of Network Flow Programming to the $\mathrm{Hy}$ drothermal Coordination in an Energy Management System” IEEE Trans. on Power Systems, vol 8, no. 3, August 1993, pp. 1045-1053.

[6] Wang, C., S.M. Shahidehpour. "Power Generation Scheduling for Multi-Area Hydro-Thermal Systems with Tie Line Constraints, Cascaded Reservoirs and Uncertain Data", IEEE Trans. on Power Systems , vol. 8, no. 3, August. 1993, pp. 1333-1340.

[7] Franco, P.E.C., M.F. Carvalho, S. Soares. "A Network Flow Model for Short-Term Hydro-Dominated Hydrothermal Scheduling Prollems". presented at the IEEE/PES 1993 Summer Meeting, Vancouver, B.C., Canada.

[8] Carvalho, M.F., S. Soares. "An efficient Hydrothermal Scheduling Algorithm". IEEE Trans. on Power Systems, vol. 2, no. 3, August 1987, pp. 537-542.

[9] Habibollahzadeh, H., G.X. Luo, A. Semlyen. "Hillrothermal Optimal Power Flow Based on Combined Linear and Nonlinear Programming Methodolory". IEEE Trans. on Power Systems, vol. 4, no. 2, May 1989, pp. 530-537.
[10] Carvalho, M.F. , S. Soares, T. Ohishi. "Optimal Power Dispatch by Network Flow Approach". IEEE Trans. on Power Systems, vol. 3, no. 4, November 1988, pp. 1640-1646.

[11] Heredia, F.J., N. Nabona. "Development and Computational Tests of an Undecoupled Optimum Short-Term Hydrothermal Scheduling Code using Network Flows". To be published in TOP, the Journal of the "Sociedad Española de Estadística e Investigación Operativa". 1994.

[12] Kennington, J.L., R.V. Helgason, "Algorithms for network programming”, John Wiley \& Sons, New York, 1980.

[13] Heredia, F.J., N. Nabona, "Numerical implementation and computational results of nonlinear network optimization with linear side constraints", in Proceedings of the 15th IFIP Conference on System Modelling and Optimization. P. Kall editor. Springer-Verlag. 1992. pp. 301-310.

[14] Heredia, F.J. "NOXCB 6.2. Manual d'usuari". Technical Report, Dept. of Statistics and Operations Research. Universitat Politècnica de Catalunya. 1991 .

[15] Murtagh, B.A. and Saunders, M.A. "A projected Lagrangian algorithm and its implementation for sparse nonlinear constraints". Mathematical Programming Study, vol. 16, 1978, pp. 84-117.

[16] Murtagh, B.A. and Saunders, M.A. "MINOS 5.0. User's guide". Dept. of Operations Research, Standford University, CA 9430, USA. 1983.

\section{BIOgRAPHIES}

F. Javier Heredia graduated from Universitat de Barcelona in 1988 with a BSc degree in Physics. Since 1989 he has been teaching and working towards a PhD degree in Operations Fesearch in the Department of Statistics \& Operations Research of the Universitat Politècnica de Catalunya, Barcelona.

Narcis Naboua (S'72-M'74) received the Energy Engineering degree from Universitat Politècnica de Catalunya (UPC) in 1968, the Certificate AEA in Control Systems from Université Paul Sabatier, Toulouse (France) in 1969, the MSc and the PhD in Electrical Engineering from the University of Alberta, Edmonton (Canada) in 1970, and from Imperial College of Science and Technology, London (G.B.) in 1974. He developed power system analysis and production planning software until 1984. Then he joined the Statistics and Operations Research Department at UPC, where he is currently Professor of Optimization. 